\title{
Control Method for Three-Phase Grid-Connected Inverter PV System Employing Unity Power Factor (UPF) Strategy in Microgrid
}

\author{
Amirreza Naderipour ${ }^{1}$, Zulkurnain Abdul-Malek ${ }^{1, *}$, Vigna K. Ramachandaramurthy ${ }^{2}$ and Josep. M. Guerrero ${ }^{3}$ \\ ${ }^{1}$ Institute of High Voltage \& High Current, School of Electrical Engineering, Faculty of Engineering, Universiti Teknologi Malaysia, \\ 81310 Johor Bahru, Malaysia \\ ${ }^{2}$ Institute of Power Engineering, Department of Electrical Power Engineering, Universiti Tenaga Nasional, Jalan Ikram-Uniten, Kajang \\ 43000, Malaysia \\ ${ }^{3}$ Institute of Energy Technology, Aalborg University, Aalborg East, Denmark, Alboorg, Denmark
}

\begin{abstract}
Microgrids (MGs) are developing owing to the rapidly growing distributed power generation systems. The MG controls the flexibility of the network to ensure the requirements of reliability and power quality are satisfied. A typical MG normally consists of dispersed generation resources, which are connected by power electronic inverters, storages, and non-linear loads. This study deals with a compensation control method of a photovoltaic grid-connected inverter using unity power factor (UPF) strategy in MG. In this case, the proposed control method can provide output currents without distortion and with the UPF. Further, it is able to increase the inverter output current to approximately 19 times of the value obtained conventionally. The proposed control method can be applied to three-phase grid interfaced converters such as DG inverters and can also be easily integrated into the conventional control scheme without installation of extra hardware. A theoretical analysis is presented and the performance of the proposed control method for a grid-connected inverter in a MG is evaluated through simulation results.
\end{abstract}

\section{Introduction}

Since the late 1980s, power quality is an important parameter for electrical companies and customers wo require high and low voltages. Consequently, electrical companies have attempted to improve the power quality in the power grid to satisfy the requests of customers. In recent times, the use of distributed generation systems (DGSs) are increasing as there is a growth in the utilisation of renewable energy sources as well as the evolution of synchronized generation units.

A distributed generation (DG) unit is a source of electrical generation that is associated either to the distribution grid directly or to customers. The microgrid (MG) distribution network consists of DG units, local loads, and energy storage, which can function in gridconnected [1] or islanded modes [2]. Based on these principles, the MG construction that was studied is illustrated in Figure 1. The photovoltaic (PV) cell, microturbine, fuel cell, and wind turbine are often intermittent. These energy systems can be combined or connected to a local energy storage system to maintain a continuous power flow between the mains grid and the local network. Even though the utilization of renewable energy sources (RESs) adds complexity to the aforementioned optimality condition, they offer various technical, economical [3], and environmental [4] advantages. Such benefits might be in the form of reducing line losses, improving voltage profiles, enhancing power quality, shaving demand peaks, increasing system reliability, and rising grid security [5]. In most cases, either a harmonic voltage or current source can represent a harmonic-producing load. Non-linear loads can produce harmonic loads and cause the system to be unbalanced and consume reactive power; further, the lack of a suitable compensator can practically worsen the power quality of the AC system.

The converter interface is often used because the output voltage of DGs is often DC power (such as, solar system or fuel cell) or AC power with variable frequencies (e.g. wind turbines) or even $\mathrm{AC}$ with a frequency that is greater than the frequency of the network (such as microturbines).

By implementing DGSs in a $\mathrm{MG}$, this situation becomes more serious, because power electronic inverters are often used to connect DG units. A MG is a local power supply system, which integrates the RESs [6], energy storage devices, and either loads or non-linear loads. The grid-connected inverters (GCIs) in DGSs and MGs are essential interfaces to connect the RESs and utility grid [7]. The power quality at the point of common coupling (PCC) will seriously influence the stability of GCIs. 


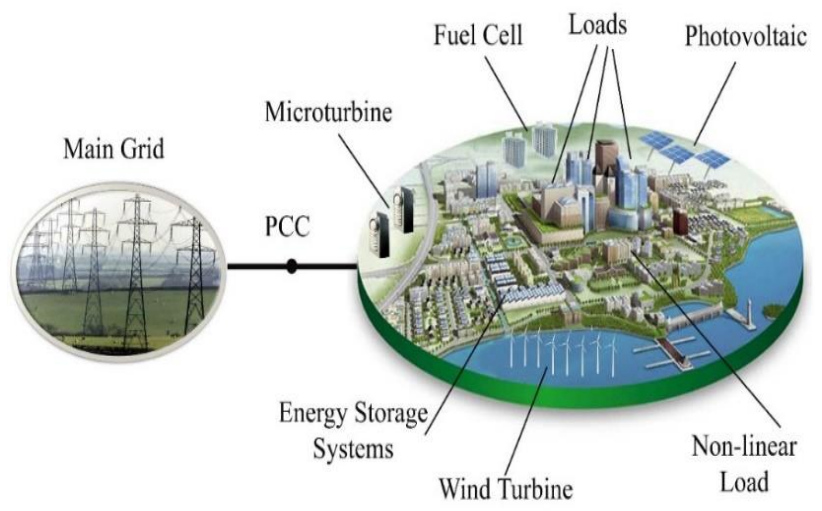

Fig. 1. Schematic of microgrid.

As a power electronic inverter may produce harmonics and require reactive power [8], a suitable compensator system will be required to maintain the quality of power. With the widespread use of harmonic generating devices, the control of harmonic currents to maintain a high level of power quality is becoming increasingly important. While facing these challenges, an effective method for harmonic suppression is the harmonic compensation achieved by using passive/active power and hybrid compensator filters. With extensive research on the harmonic pollution of the power grid in the early 1990s, a new approach concerning harmonic compensation in the power system was offered [9].

To reduce the investment, operation, and maintenance costs, and increase the cost-effective feature of the GCIs in DGSs and MGs, new control methods for GCIs were proposed [10], [11]. The non-linear loads are capable of creating harmonics and can result in an unstable system that would consume reactive power. Furthermore, frequently, the lack of a suitable compensator may deteriorate the power quality of the AC system.

By implementing the DG in a MG, this situation becomes more serious as power electronic inverters are frequently used with the aim to connect DG units such as fuel cells, wind turbines. Further, the power electronic inverter might produce harmonics and the prerequisite reactive power. Moreover, an appropriate compensator system will be required to retain the power quality.

This paper presents a compensation control method for the PV GCI using unity power factor (UPF) strategy in the MG. The performance of the proposed control is demonstrated using computer simulations and verified using a real control method for a GCI in the MG for simultaneous reference power injection and harmonics rejection.

\section{Proposed control method}

In this proposed control method, the load and GCI are observed through the source by means of a linear resistance. After applying the UPF condition, the voltage and current sources at the PCC will have indiscernible waves by the different domains (e.g. have equal total harmonic distortion (THD) values) [12]. At this stage, the reference source current given by

$$
i_{s}=k v_{p c c}
$$

where $k$ is a constant. At that time, the power can be delivered by the source at the PCC, which can be written as

$$
p_{s}=\vec{v}_{p c c} \vec{i}_{s}=\vec{v}_{p c c} \cdot k v_{p c c}=k v_{(0 \alpha \beta)}^{2}
$$

If we consider that the power delivered by the source is equal to the DC component of the load instantaneous active power; then

$$
k=\frac{\bar{p}_{L \alpha \beta}+\bar{p}_{L 0}}{v_{0 \alpha \beta}^{2}}
$$

Therefore, the reference values of the source current can be determined by simple calculations, i.e.

$$
\left[\begin{array}{c}
i_{s 0} \\
i_{s \alpha} \\
i_{s \beta}
\end{array}\right]=k\left[\begin{array}{c}
v_{0} \\
v_{\alpha} \\
v_{\beta}
\end{array}\right]=\frac{\bar{p}_{L \alpha \beta}+\bar{p}_{L 0}}{v_{0 \alpha \beta}^{2}}\left[\begin{array}{c}
v_{0} \\
v_{\alpha} \\
v_{\beta}
\end{array}\right]
$$

Finally, the neutral source current, is given by [13].

$$
i_{n S}(t)=\sqrt{3} \cdot i_{s 0}(t)=\frac{\sqrt{3} \bar{p}_{L}(t)+e_{0}(t)}{\left(e_{0 \alpha \beta}^{2}\right)_{d c}}
$$

To obtain the current set point for each phase, the output of the proportional-integral (PI) controller is multiplied with the mains voltage waveform. Figure 2 shows that the reference currents of UPF for the PWM can be achieved by the PI output voltage controller. Hence, the limiter is responsible for providing the shortcircuit-proof operation.

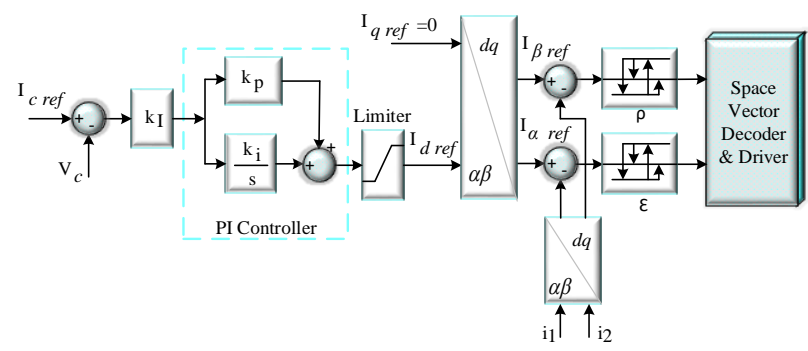

Fig. 2. Proportional-integral output voltage controller.

The reference $i_{\text {qref }}$ for the $i_{q}$ current is derived from the desired power factor. In Figure $3, R$ and $C$ are resistance and the value of the output capacitor, respectively. 


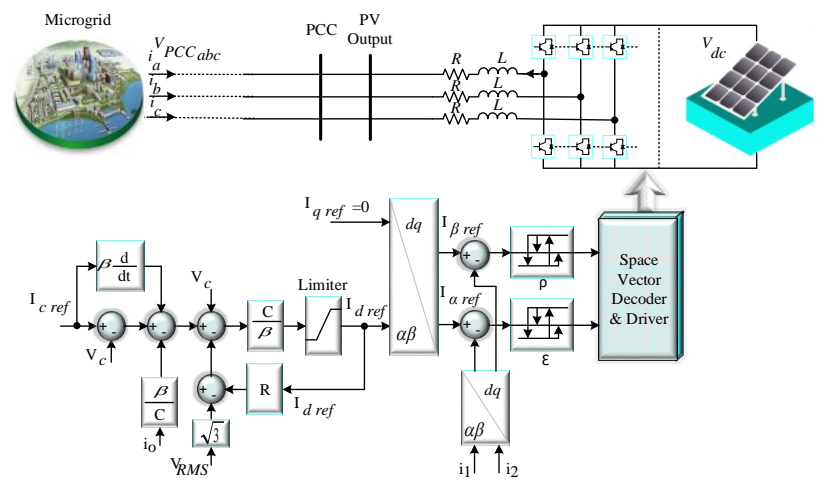

Fig. 3. Proposed control method for the unity power factor three-phase PWM.

The load current $i_{o}$ is considered as a time-dependent perturbation. The $i_{d}$ and $i_{q}$ currents usually exhibit very fast dynamics when compared to $V c$. Therefore, using three autonomous two-level hysteresis comparators can accomplish the practical implementation of the switching strategy. Hence, the control laws can be performed by the setup shown in the block diagram illustrated in Figure 3.

\section{Simulation results}

Figure 4 shows the simulation circuit to confirm the performance of the suggested non-linear loads and PV cell in $\mathrm{MG}$, which was simulated using the MATLAB/Simulink software.

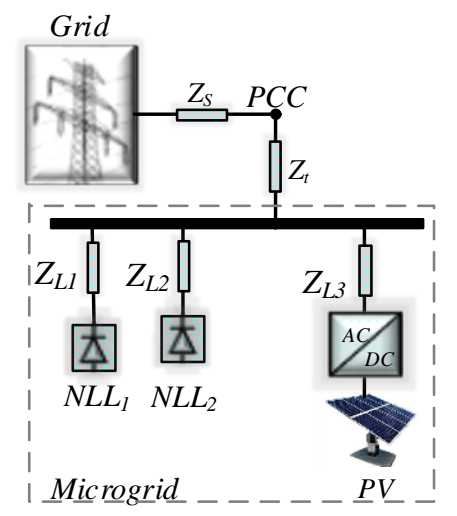

Fig. 4. Study system configuration with photovoltaic (PV) cell.

While considering the above-mentioned problems, it is necessary to develop and use effective power quality improvement facilities to reduce the harmonics content of system current, compensate reactive power, and increase the power quality of the distribution systems. Hence, Figure 4 shows the configuration of the analysed system. As it can be observed, the PV cell and two non-linear loads are implemented to construct a sample distribution network. In this study, the PV cell was used as a DG unit; consequently, all the mentioned units (non-linear load 1, non-linear load 2, and PV cell) were considered as current sources which inject current harmonics to the system. A linear relation was assumed between the inductance and frequency. The PV cell is connected to the MG by the three-phase GCI. The parameters of the three-phase power line are listed in Table 1.

Table 1. Power Line Parameters

\begin{tabular}{|c|c|c|c|c|c|}
\hline Parameters & $\mathbf{Z L}_{\mathbf{1}}$ & $\mathbf{Z L}_{\mathbf{2}}$ & $\mathbf{Z L}_{\mathbf{3}}$ & $\mathbf{Z}_{\mathbf{t}}$ & $\mathbf{Z}_{\mathbf{s}}$ \\
\hline $\mathrm{R}(\Omega)$ & 0.53 & 0.29 & 0.14 & - & - \\
\hline $\mathrm{L}(\mathrm{mH})$ & 1.31 & 0.67 & 0.31 & 0.16 & 0.01 \\
\hline
\end{tabular}

The current waveforms of the uncompensated grid, PV cell, non-linear loads 1 and 2 are shown in Figures 5 (a)-(d). These figures show the currents, equipped without any compensation, which is connected to the MG. The currents are measured at 188.4 (A), 47.76 (A), 24.4 (A), and 117.7 (A) for the uncompensated grid, PV cell, non-linear loads 1 and 2, respectively, and the THDs are measured at $12.67 \%, 8.01 \%, 7.23 \%$, and $17.32 \%$, respectively.

Thus, certain limited solutions were proposed for imbalance and current harmonics in different standards. For instance, the International Electrotechnical Commission suggests that the current imbalance of electrical systems should be below 2\% [13]. Additionally, according to the IEEE standard (No. 519-1992), the maximum allowable current distortion in each of the harmonics and the THD is 3\% and 5\%, respectively [14].

Although a separate standard has not been provided for power quality indicators in MGs, there could be sensitive loads in a MG that require higher power quality. Therefore, the maximum permissible distortion values for MGs should be lower. Considering this, it is essential to note that one of the main objectives for the deployment of DGSs, especially on the MG structure, is to provide reliable and high-quality power.

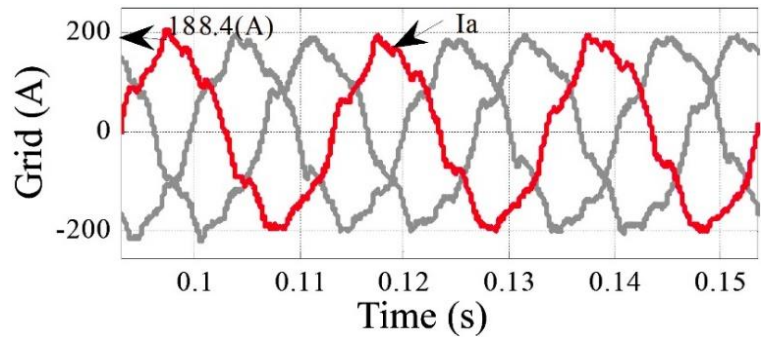

(a)

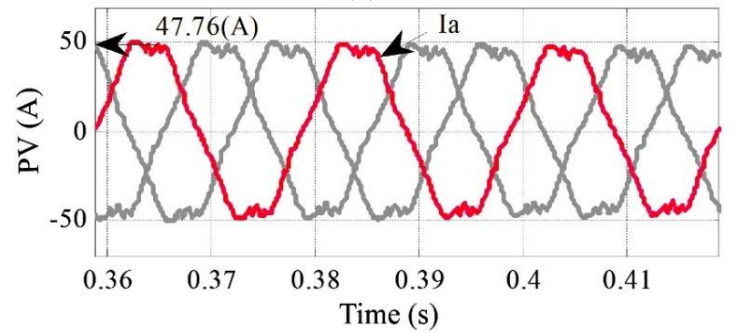

(b)

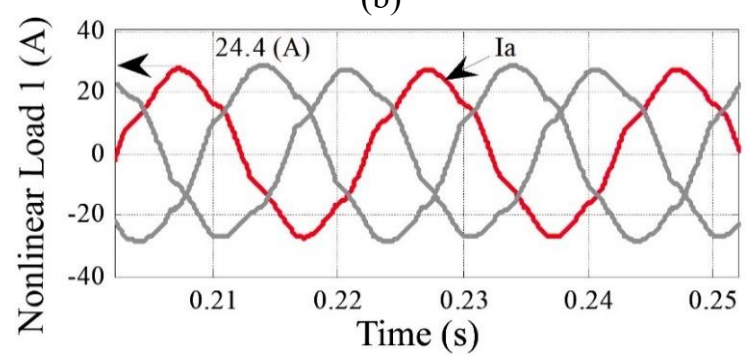

(c) 


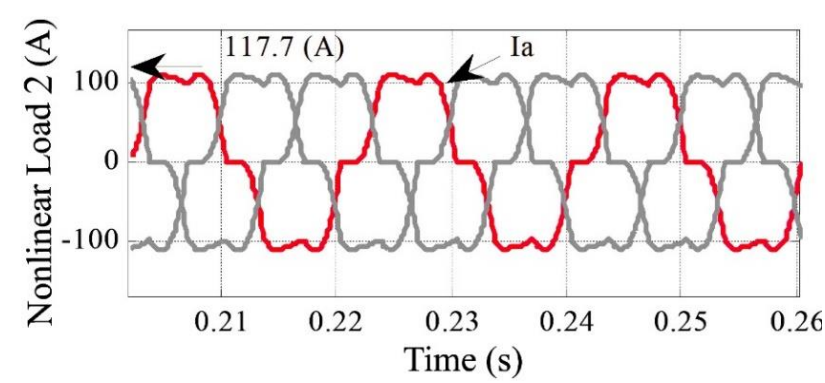

(d)

Fig. 5. Distortion current waveforms without compensation: (a) grid current; (b) PV current; (c) non-linear load 1 current and (d) non-linear load 2 current.

Subsequently, the research demonstrated an improved power quality using the proposed control method. The compensated system currents are explained in this subsection. Figures 6 (a) and (b) show the current waveforms of the system and PV cell. The THDs are $1.02 \%$ and $0.87 \%$, respectively. Figures 6 (a) and (b) show the effective compensation values of the harmonic current for the system and PV cell.

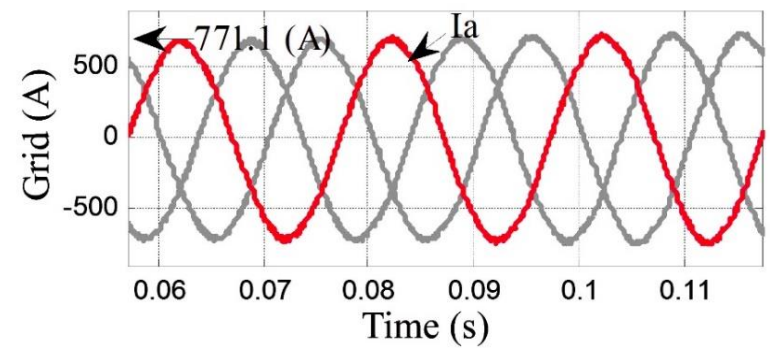

(c)

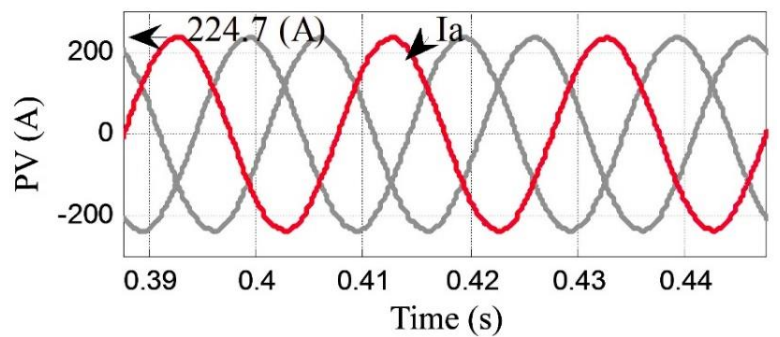

(d)

Fig. 6. Grid and distributed generation unit current waveforms with proposed control method; (a) system currents; (b) PV cell.

The current and THD values before and after the proposed control method are listed in Table 2 . This case shows that the proposed control method can compensate for the current system and PV cell.

Table 1. Current and THD Results

\begin{tabular}{|c|c|c|c|c|}
\hline \multirow{2}{*}{} & \multicolumn{2}{|c|}{$\begin{array}{c}\text { Before } \\
\text { Compensation }\end{array}$} & \multicolumn{2}{c|}{$\begin{array}{c}\text { After Propose } \\
\text { Control Method }\end{array}$} \\
\cline { 2 - 5 } & $\begin{array}{c}\text { Current } \\
\text { (A) }\end{array}$ & $\begin{array}{c}\text { THD } \\
\text { \% }\end{array}$ & $\begin{array}{c}\text { Current } \\
\text { (A) }\end{array}$ & $\begin{array}{c}\text { THD } \\
\text { \% }\end{array}$ \\
\hline Grid & 188.4 & 12.67 & 771.1 & 1.02 \\
\hline PV & 47.76 & 8.01 & 224.7 & 0.87 \\
\hline N-load1 & 24.4 & 7.23 & - & - \\
\hline N-Load2 & 117.7 & 17.32 & - & - \\
\hline
\end{tabular}

\section{Conclusion}

One of the power quality problems in MGs is the THD of the interface inverter for the current exchanged with the grid. GCIs are effective interfaces for DGSs. This paper presented a new control strategy for harmonic current compensation for the PV GCI in an MG. The proposed control method consisted of an advance UPF strategy. Simulation studies were performed for a power system that included a PV cell connected into the system through GCI and non-linear loads, which produced the distorted waveforms. The proposed control method could transfer or compensate unbalanced currents and harmonic currents simultaneously. Finally, simulation results were provided to verify the effectiveness and performance of the proposed control method on PV GCI in MG.

\section{Acknowledgement}

The authors wish to thank the Ministry of Education (MOE) and Universiti Teknologi Malaysia for the financial support under the Post-Doctoral Fellowship Scheme (grant numbers 4F828, 04E54, and 18H10) for the financial support.

\section{References}

1. A. A. M. Zin, A. Naderipour, M. H. Habibuddin, and J. M. Guerrero, "Harmonic currents compensator GCI at the microgrid," Electron. Lett., 2016.

2. A. Naderipour, Z. Abdul-Malek, V. K. Ramachandaramurthy, A. Kalam, and M. R. Miveh, "Hierarchical control strategy for a three-phase 4wire microgrid under unbalanced and nonlinear load conditions," ISA Trans., 2019.

3. R. K. Singh and S. K. Goswami, "Optimum allocation of distributed generations based on nodal pricing for profit, loss reduction, and voltage improvement including voltage rise issue," Int. J. Electr. Power Energy Syst., vol. 32, no. 6, pp. 637644, 2010.

4. P. D. C. Wijayatunga, W. Fernando, and R. M. Shrestha, "Impact of distributed and independent power generation on greenhouse gas emissions: Sri Lanka," Energy Convers. Manag., vol. 45, no. 20, pp. 3193-3206, 2004.

5. S. Ghosh, S. P. Ghoshal, and S. Ghosh, "Two analytical approaches for optimal placement of distributed generation unit in power systems," in Power Systems, 2009. ICPS'09. International Conference on, 2009, pp. 1-6.

6. D. Wu, F. Tang, T. Dragicevic, J. C. Vasquez, and J. M. Guerrero, "A control architecture to coordinate renewable energy sources and energy storage systems in islanded microgrids," Smart Grid, IEEE Trans., vol. 6, no. 3, pp. 1156-1166, 2015.

7. Z. Zeng, H. Yang, R. Zhao, and C. Cheng, "Topologies and control strategies of multifunctional grid-connected inverters for power quality 
enhancement: A comprehensive review," Renew. Sustain. Energy Rev., vol. 24, pp. 223-270, 2013.

8. M. Ranjbar, S. Mohaghegh, M. Salehifar, H. Ebrahimirad, and A. Ghaleh, "Power electronic interface in a $70 \mathrm{~kW}$ microturbine-based distributed generation," in Power Electronics, Drive Systems and Technologies Conference (PEDSTC), 2011 2nd, 2011, pp. 111-116.

9. S. A. Moran and M. B. Brennen, "Active power line conditioner with fundamental negative sequence compensation." Google Patents, 24-Jan-1995.

10. G. Tsengenes and G. Adamidis, "Investigation of the behavior of a three phase grid-connected photovoltaic system to control active and reactive power," Electr. Power Syst. Res., vol. 81, no. 1, pp. 177-184, 2011.
11. M. Triggianese, F. Liccardo, and P. Marino, "Ancillary services performed by distributed generation in grid integration," in Clean Electrical Power, 2007. ICCEP'07. International Conference on, 2007, pp. 164-170.

12. M. Ranjbar, M. A. S. Masoum, and A. Jalilian, "Comparison of compensation strategies for shunt active power filter control in unbalanced tree-phase four-wire systems," in Electrical and Computer Engineering, 2009. CCECE'09. Canadian Conference on, 2009, pp. 1061-1066.

13. A. Von Jouanne and B. Banerjee, "Assessment of voltage unbalance," IEEE Trans. power Deliv., vol. 16, no. 4, pp. 782-790, 2001.

14. I. F II, "IEEE recommended practices and requirements for harmonic control in electrical power systems," pp. 519-1992, 1993. 